\title{
A Flexible Modulation Scheme Design for C-Band GNSS Signals
}

\author{
Rui Xue, Qing-ming Cao, and Qiang Wei \\ College of Information \& Communication Engineering, Harbin Engineering University, Harbin 150001, China
}

Correspondence should be addressed to Rui Xue; xuerui0216@hotmail.com

Received 9 February 2015; Revised 10 June 2015; Accepted 10 June 2015

Academic Editor: Emiliano Mucchi

Copyright (C) 2015 Rui Xue et al. This is an open access article distributed under the Creative Commons Attribution License, which permits unrestricted use, distribution, and reproduction in any medium, provided the original work is properly cited.

\begin{abstract}
Due to the spectrum congestion of current navigation signals in L-band, C-band has been taken into consideration as a candidate frequency band for global navigation satellite system (GNSS). As is known, modulation scheme is the core part of signal structure, and how to design a modulation waveform that could make full use of narrow bandwidth $20 \mathrm{MHz}$ and satisfy the constraint condition of frequency compatibility in C-band is the main research content of this paper. In view of transmission characteristics and constraint condition of compatibility in C-band, multi- $h$ continuous phase modulation (CPM) is proposed as a candidate modulation scheme. Then the classical channel capacity estimation and a comprehensive evaluation criterion for GNSS modulation signals are employed to assess the proposed scheme in the aspects of the capacity over additive white Gaussian noise (AWGN), tracking accuracy, multipath mitigation, antijamming, and so on. Simulation results reveal that, through optimizing the number and size of modulation indexes, the flexible scheme could offer better performance in terms of code tracking, multipath mitigation, and antijamming compared with other candidates such as MSK and GMSK while maintaining high band efficiency and moderate implementation complexity of receiver. Moreover, this paper also provides a reference for next generation modulation signals in C-band.
\end{abstract}

\section{Introduction}

With the rapid development of global navigation satellite systems (GNSS), namely, GPS, GLONASS, Galileo, and Compass, together with various regional navigation satellite systems and space-based augmentation systems, the number of navigation signals in space is anticipated to be over 400 by 2030 [1], which will further aggravate an already crowded radio spectrum in L-band $(1164 \sim 1610 \mathrm{MHz})$. Consequently, improving the signal spectral efficiency [2] and providing new bands for radio navigation satellite service (RNSS) are the main means to solve the above problem, and the frequency band between $5010 \mathrm{MHz}$ and $5030 \mathrm{MHz}$ offering a bandwidth of $20 \mathrm{MHz}$ has been already allocated as C-band portion by International Telecommunication Union (ITU) for RNSS applications. Unfortunately, the use of C-band navigation signals offers both advantages and drawbacks [3]. However, technological progress in electronics and spacecraft might balance some of the disadvantages, and C-band has caused great interests as a candidate for the future GNSS services [4].
Although the performance of signals in C-band is difficult to surpass L-band [5], signal combination of L- and Cband could improve positioning accuracy and timing performance and promote the comprehensive performance of RNSS $[6,7]$. Predictably, the multiband combined navigation and compatibility among different navigation systems will become research hotspots in the future. As is known to all, modulation scheme whose quality completely determines the upper bound of GNSS performance is the core part of signal structure, and power spectrum envelopes decided by modulation waveforms could play a dominant role in tracking accuracy, multipath mitigation, antijamming, and so forth. At present, how to design a modulation scheme that can make full use of bandwidth $20 \mathrm{MHz}$ and satisfy the constraint condition of frequency compatibility is the main challenge subject of C-band signal system.

The compatibility between newly introduced signals in C-band and the existing signals in adjacent frequency bands such as radio astronomy (RA) band and microwave landing system (MLS) must be carefully analyzed before any new band for satellite navigation is put into use [8]. 
Therefore, band-limited signals or signals with continuous phase should be the first choice for C-band signal system. In addition, the signals are easily distorted due to the nonlinear effect of the high power amplifier (HPA) [9], and a constant envelope modulation reveals the excellent characteristic that HPA can run in saturation or close to saturation, which could increase the total efficiency of amplification. By the above analysis, a modulation scheme with constant envelope and continuous phase will be a top priority for C-band signal.

Binary offset carrier (BOC) modulation and BOCderived modulations such as multiplexed BOC (MBOC) and alternative BOC (AltBOC) have been already widely accepted by next generation GNSS. Nevertheless, the BOC modulated signals have larger spectral side lobes and are more prone to produce larger interferences with signals of other coexisting navigation systems [10]. Reference [11] has pointed out that minimum shift keying (MSK) can offer better ranging precision within a certain bandwidth and introduce less interference to other signals. Subsequently, MSK, MSK-BOC, and Gaussian-filtered MSK (GMSK) have been proposed as potential candidates for C-band signals by Galileo system. However, the tracking bias of MSK-BOC modulated signals has not been solved effectively so far [12], and hardware implementation of GMSK is complicated and its tracking performance is hard to optimize. Moreover, MSK and GMSK are the special cases in continuous phase modulation (CPM), but they do not possess the optimal performance in CPM family.

Based on the above problems, a special subclass of CPM, that is, multi- $h$ CPM, is presented as a modulation waveform for C-band signal due to its many excellent characteristics, such as flexible parameter adjusting, high power and spectrum efficiency, a large number of alternative waveforms, and being easily compatible with existing signals. The rest of this paper is organized as follows. Section 2 describes mathematical model and an improved calculation of power spectra for multi- $h$ CPM signals. The capacity of multi$h$ CPM signals is derived in Section 3. Section 4 provides performance evaluation criterion for GNSS modulation. Simulation results are discussed in Section 5. Finally, we conclude the paper in Section 6.

\section{Multi- $h$ CPM Signal}

2.1. Mathematical Model. The complex-baseband multi- $h$ $\mathrm{CPM}$ signal is given by

$$
s(t ; \boldsymbol{\alpha})=\exp \{j \phi(t ; \boldsymbol{\alpha})\},
$$

where $\phi(t ; \boldsymbol{\alpha})$ is the information-carrying phase, denoted as

$$
\phi(t ; \boldsymbol{\alpha})=2 \pi \sum_{i=-\infty}^{\infty} \alpha_{i} h_{\underline{i}} q(t-i T),
$$

where $T$ is the symbol duration, $\boldsymbol{\alpha}=\left\{\alpha_{i}\right\}$ are the information symbols in the $M$-ary alphabet $\{ \pm 1, \pm 3, \ldots, \pm(M-1)\}, q(t)$ is the phase pulse, and $\left\{h_{i}\right\}_{i=0}^{N_{h}-1}$ is a set of $N_{h}$ modulation index where $h_{i}$ is constant in symbol duration $T$. In this paper, the underlined subscript notation in (2) is defined as modulo- $N_{h}$; that is, $\underline{n} \triangleq n \bmod N_{h}$. We always assume $h_{i} \leq 1$ because power spectra of CPM will behave like BOC signals with spectrum splitting when modulation index is more than one, which goes against compatibility with other signals in relatively narrow bandwidth.

The phase pulse $q(t)$ and the frequency pulse $f(t)$ are related by

$$
q(t)=\int_{-\infty}^{t} f(\tau) d \tau .
$$

The frequency pulse is supported over the time interval $(0$, $L T)$ and is subject to the constraints

$$
\int_{0}^{L T} f(\tau) d \tau=q(L T)=\frac{1}{2} .
$$

When $L=1$ the signal is called full-response formats and when $L>1$ the signal is called partial-response formats. Some general pulse shapes are length-LT rectangular (LREC), length- $L T$ raised-cosine (LRC), and Gaussian.

In light of the constraints on $f(t)$ and $q(t),(2)$ can be rewritten as

$$
\begin{aligned}
\phi(t ; \boldsymbol{\alpha})= & \theta\left(t ; \boldsymbol{\alpha}_{n}\right)+\theta_{n-L} \\
= & 2 \pi \sum_{i=n-L+1}^{n} \alpha_{i} h_{\underline{i}} q(t-i T) \\
& +\left(\pi \sum_{i=-\infty}^{n-L} \alpha_{i} h_{\underline{i}}\right) \bmod 2 \pi,
\end{aligned}
$$

where the term $\theta\left(t ; \boldsymbol{\alpha}_{n}\right)$ is a function of the $L$ symbols being modulated by the phase pulse. For $h_{\underline{i}}=2 k_{\underline{i}} / p\left(k_{\underline{i}}, p\right.$ integers $)$, the phase state $\theta_{n-L}$ takes on $p$ distinct values. The signal is described by a trellis containing $p M^{L-1}$ states, with $M$ branches at each state. Each branch is defined by the $(L+1)$ tuple $\sigma_{n}=\left(\theta_{n-L}, \alpha_{n-L+1}, \alpha_{n-L+2}, \ldots, \alpha_{n}\right)$.

2.2. Easy Calculation of Power Spectra. Reference [13] provides an easy method which allows a fast calculation of the spectra of $M$-ary multi- $h$ CPM signals under the general hypothesis of (1) $M$-ary symbols ( $M$ a power of 2); (2) arbitrary pulse shaping; (3) partial response signaling; (4) an arbitrary set of modulation indexes. The procedure of the easy method is as follows.

Let $\tilde{x}(t)=\exp \{j \phi(t)\}$ be the complex envelope of the transmitted signal $x(t)$. To find the baseband equivalent $\widetilde{S}(f)$ of the power spectrum $S(f)$ of the signal $x(t)$, we use the socalled "autocorrelation-based" approach.

The autocorrelation function of $\tilde{x}(t)$ is defined as

$$
\begin{aligned}
& \rho\left(t_{1}, t_{2}\right) \\
& \simeq E\left\{\tilde{x}\left(t_{1}\right) \tilde{x}^{*}\left(t_{2}\right)\right\} \\
& =E\left\{\exp \left[j \sum_{i=-\infty}^{+\infty} 2 \pi \alpha_{i} h_{i}\left(q\left(t_{1}-i T\right)-q\left(t_{2}-i T\right)\right)\right]\right\} .
\end{aligned}
$$


As we know, $h_{i+K}=h_{i}$ and $K T=$ superbaud period and the independence of the data symbols; we are able to put $\rho\left(t_{1}, t_{2}\right)$ in the form; that is,

$$
\begin{aligned}
& \rho\left(t_{1}, t_{2}\right) \\
& =\prod_{i=-\infty}^{+\infty} \sum_{r=0}^{M-1} \frac{1}{M} \exp \left\{j 2 \pi(2 r-M+1) h_{\underline{i}} \times\left[q\left(t_{1}-i T\right)-q\left(t_{2}-i T\right)\right]\right\} \\
& =\prod_{i=-\infty}^{+\infty} p\left(t_{1}-i K T, t_{2}-i K T\right),
\end{aligned}
$$

where

$$
p(a, b) \simeq \prod_{m=0}^{K-1} \frac{\sin \left(2 \pi M h_{m}(q(a)-q(b))\right)}{M \sin \left(2 \pi h_{m}(q(a)-q(b))\right)} .
$$

The process $\tilde{x}(t)$ is cyclostationary in the wide sense with period $K T$ and average autocorrelation is expressed as

$$
\widetilde{R}(\tau) \simeq \frac{1}{K T} \int_{0}^{K T} \rho(t, t+\tau) d t .
$$

It is seen that $p(a, b)=1$ when $a, b \geq(L+K-1) T$ or $a, b \leq 0$. The number of factors in (7) can be reduced and (9) is rewritten as

$$
\begin{aligned}
& \widetilde{R}(\tau)=\frac{1}{K T} \int_{0}^{K T} \prod_{i=\lceil(1-L) / K\rceil}^{m+1} p\left(t-i K T, t+\tau^{\prime}\right. \\
& -(i-m) K T) d t
\end{aligned}
$$

where $m \simeq\lfloor\tau / K T\rfloor, \tau^{\prime} \simeq \tau-m K T\left(0 \leq \tau^{\prime}<K T\right),\lfloor y\rfloor \simeq$ maximum integer $\leq y$, and $\lceil y\rceil \simeq$ minimum integer $\geq y$.

The power spectral density (PSD) $\widetilde{S}(f)$ can be derived from $\widetilde{R}(\tau)$ by Fourier transform (FT); that is,

$$
\begin{aligned}
& \widetilde{S}(f)=2\left\{\int_{0}^{(1-s) K T} \widetilde{R}(\tau) \cos 2 \pi f \tau d \tau\right. \\
&\left.+\mathbf{R}\left[\frac{\int_{(1-s) K T}^{(2-s) K T} \widetilde{R}(\tau) \exp (-j 2 \pi f \tau) d \tau}{\left(1-C_{1, K} \exp (-j 2 \pi f K T)\right)}\right]\right\},
\end{aligned}
$$

where $s \simeq\lfloor(1-L) / K\rfloor, C_{1, K} \simeq \prod_{m=0}^{K-1}\left[\sin \left(M \pi h_{m}\right) /\right.$ $\left(M \sin \left(\pi h_{m}\right)\right)$ ], and $\mathbf{R}(\cdot)$ denotes real part of complex.

In this paper, we always assume the spread spectrum code rate and $M$ in all schemes are equal to $5 \times 1.023 \mathrm{MHz}$ and 2, respectively. The PSD of CPM signals with different parameters are shown in Figure 1. As seen in Figure 1, the CPM signals using RC pulse can effectively decrease PSD amplitude of side lobes and concentrate more energy into main lobe compared to REC pulse when the modulation indexes are the same. Moreover, it is noteworthy that energy in main lobe tends to be more centralized and decay rate of side lobes is almost the same when the average of all elements in set $\left\{h_{1}, h_{2}, \ldots\right\}$ is getting larger. In terms of main lobe energy, the CPM signals with RC or REC are more concentrated than GMSK. Also, power fluctuation of side

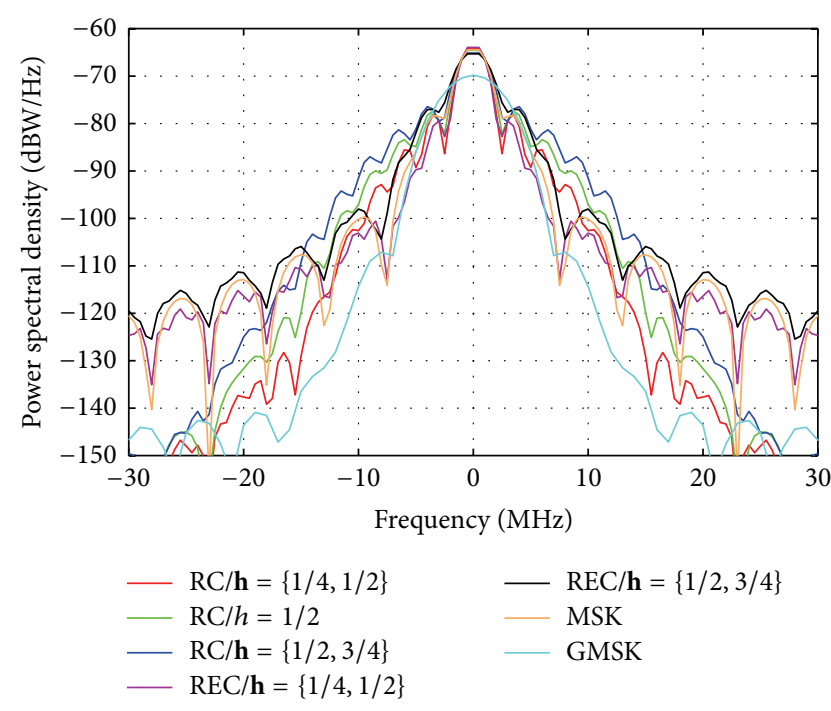

FIgURE 1: The PSD of different CPM signals.

lobes in GMSK decays faster than CPM signals with REC, inferior to CPM adopting RC pulse.

The PSD of modulated signals have a direct effect on tracking performance, ability of multipath mitigation, and antijamming. Particularly, the PSD of modulated signals should have the following characteristics in order to meet the strict compatibility condition constraints of the C-band: (1) the majority of power should be concentrated into the main lobe; (2) a stronger spectrum roll-off in side lobes should be provided in order to significantly cut down spectrum leakage or interference to RA and MLS services.

In the CPM family, multi- $h$ CPM signals as a special subclass still maintain all of excellent properties just as a general CPM. In addition, a properly chosen cyclic set of modulation indexes results in delayed merging of neighboring phase trellis paths and, therefore, provides a larger minimum Euclidean distance than conventional single- $h$ CPM, which leads to improving error performance and making its spectrum more compact, and out-of-band rejection better. Under the condition of limited bandwidth and power, just as satellite navigation systems, multi- $h$ CPM has more excellent transmission performance than single- $h$ CPM [14]. By the above analysis, "flexible" means that a particular multi- $h$ $\mathrm{CPM}$ can be designed according to some restrictions in some practical systems through optimizing the number and size of modulation indexes.

\section{Capacity of CPM over AWGN Channel}

Modern satellite navigation systems employ high efficient channel codes which operate very close to capacity. This makes the channel capacity an effective parameter in evaluating the performance of a modulation scheme. It has been shown that a CPM modulator can be decomposed into a continuous phase encoder (CPE) followed by a memoryless modulator (MM). Since the CPE is Markov, the CPM modulator can be described by a finite-state machine (FSM) with 


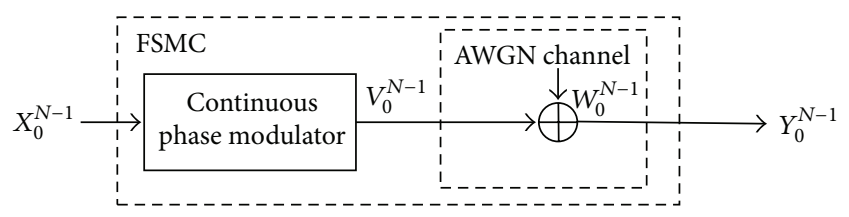

FIGURE 2: FSMC model for CPM over AWGN channel.

$p M^{L-1}$ states, which is ergodic and stationary. Thus the CPM modulator together with AWGN channel can be modeled as a FSM channel (FSMC) shown in Figure 2, and the capacity of $\mathrm{CPM}$ is the mutual information between the input and output sequences of FSMC [15].

Suppose $\left(X_{0}, X_{1}, \ldots, X_{N-1}\right)$ is the transmitted symbol sequence and $\left(Y_{0}, Y_{1}, \ldots, Y_{N-1}\right)$ is the received sequence of symbols. Let $X_{i}^{j}$ denote the sequence of symbols $X_{i}$, $X_{i+1}, \ldots, X_{j}$. Let $I\left(X_{0}^{N-1} ; Y_{0}^{N-1}\right)$ denote the mutual information between the transmitted and the received sequences. Then, the channel capacity can be calculated as

$$
C_{\text {i.u.d }}=\lim _{N \rightarrow \infty} \frac{1}{N} I\left(X_{0}^{N-1} ; Y_{0}^{N-1}\right) \text {. }
$$

From the chain rule of entropy

$$
\begin{aligned}
I\left(X_{0}^{N-1} ; Y_{0}^{N-1}\right)= & H\left(X_{0}^{N-1}\right)-H\left(X_{0}^{N-1} \mid Y_{0}^{N-1}\right) \\
= & \sum_{i=0}^{N-1} H\left(X_{i}\right) \\
& -\sum_{i=0}^{N-1} H\left(X_{i} \mid X_{0}^{i-1}, Y_{0}^{N-1}\right)
\end{aligned}
$$

where $H\left(X_{i} \mid X_{0}^{i-1}\right)=H\left(X_{i}\right)$ is used. Because input $X_{i}$ is independent and uniformly distributed (i.u.d) over $M$ constellation points, $H\left(X_{i}\right)=\log _{2} M$, and all that remains to be calculated is $H\left(X_{i} \mid X_{0}^{i-1}, Y_{0}^{N-1}\right)$. From the definition of conditional entropy,

$$
H\left(X_{i} \mid X_{0}^{i-1}, Y_{0}^{N-1}\right)=-E\left[\log _{2} p\left(X_{i} \mid X_{0}^{i-1}, Y_{0}^{N-1}\right)\right] .
$$

The above expectation can be found using Monte Carlo integration. To compute the probability $p\left(X_{i} \mid X_{0}^{i-1}, Y_{0}^{N-1}\right)$, we first apply Bayes' rule to obtain the following equation:

$$
p\left(X_{i} \mid X_{0}^{i-1}, Y_{0}^{N-1}\right)=\frac{p\left(X_{i}, X_{0}^{N-1}, Y_{0}^{N-1}\right)}{\sum_{X_{i}} p\left(X_{i}, X_{0}^{i-1}, Y_{0}^{N-1}\right)}
$$

Similar to [16], a BCJR-like method can be used to compute $p\left(X_{i}, X_{0}^{N-1}, Y_{0}^{N-1}\right)$. Rather than explicitly calculating the denominator in (15), its value is found such that $\sum_{X_{i}} p\left(X_{i} \mid\right.$ $\left.X_{0}^{i-1}, Y_{0}^{N-1}\right)=1$. More details of calculation process for $p\left(X_{i}\right.$, $X_{0}^{N-1}, Y_{0}^{N-1}$ ) are shown in [17].

It is obvious that approximate estimation of channel capacity is derived from (12) (14), and computational complexity of (14) is reduced from exponential order of $N$ to linear due to BCJR introduced into Arnold algorithm.

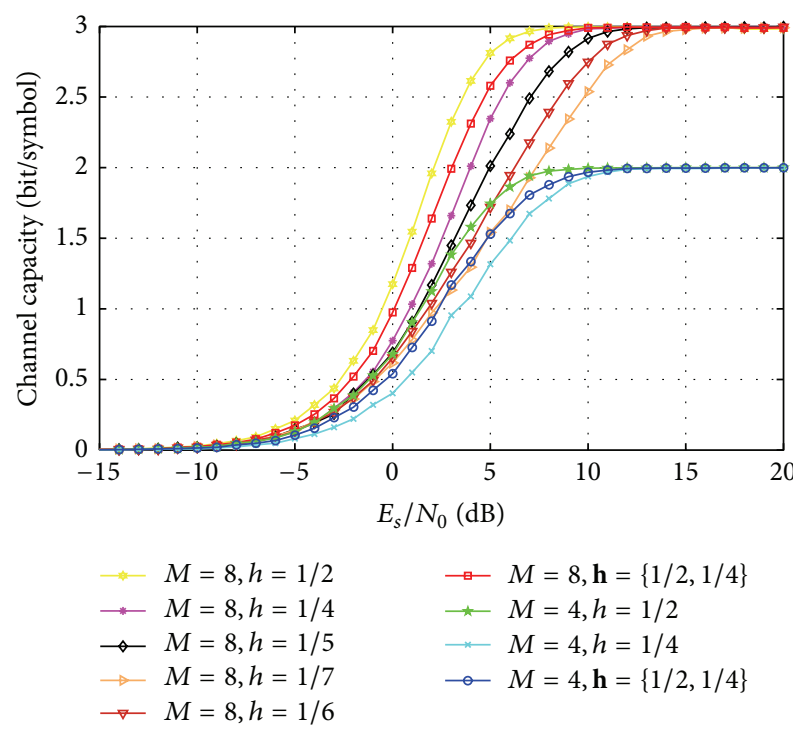

Figure 3: The channel capacity of different CPM signals.

According to Monte Carlo theory, the approximate estimation of channel capacity is infinite approaching to true value when length of $N$ is closing to infinite. In fact, the deviation of channel capacity descends with the increasing of $N$ and tends to be stable when $N$ is larger than 5000 .

Figure 3 shows the channel capacity of different CPM signals with $2 \mathrm{RC}$. We can see from Figure 3 that capacity is gradually improved with the increasing of $M$ when modulation index is fixed at a certain value. Under the same condition of $M$, the greater average of all elements in set $\left\{h_{i}\right\}$ is, the earlier channel capacity converges. That is to say, $E_{s} / N_{0}$ required to achieve the same capacity is getting smaller with the increasing of average of all elements in set $\left\{h_{i}\right\}$ if other conditions are the same. For instance, the channel capacity of signal with $\mathbf{h}=\{1 / 2,1 / 4\}$ has to be intermediate between $h=1 / 2$ and $h=1 / 4$ when $M$ is the same.

\section{Performance Evaluation for Modulation Signal}

Generally, an excellent performance evaluation criterion for modulation signal is characterized by objectivity, accuracy, comprehensiveness, adaptability, and so forth. A rather comprehensive performance evaluation method for GNSS modulation has been proposed by [18], and the tracking accuracy, multipath mitigation, and antijamming performance are used as performance evaluation standard, which provides significant references on satellite navigation signal design. Next, we will expound the above performance indexes one by one.

4.1. Tracking Accuracy. Gabor bandwidth and code tracking errors can be used as the reference indexes for evaluating the tracking performance. Based on a coherent early-minus-late 
(EML) code tracking loop, the code tracking errors in AWGN channel are defined as follows [19]:

$$
\sigma_{\varepsilon}^{2}=\frac{B_{L}\left(1-0.5 B_{L} T_{i}\right) \int_{-B / 2}^{B / 2} G(f) \sin ^{2}(\pi f d) d f}{(2 \pi)^{2}\left(C / N_{0}\right)\left[\int_{-B / 2}^{B / 2} f G(f) \sin (\pi f d) d f\right]^{2}},
$$

where $B_{L}$ denotes the tracking loop bandwidth, $G(f)$ is the PSD of the signal that is normalized to unit power over infinite transmission bandwidth and symmetric to the carrier frequency, $d$ denotes the correlation time spacing between the early and late reference signals, $C / N_{0}$ is the carrier-to-noise ratio (CNR), $B$ is the receiver prefiltering bandwidth, and $T_{i}$ is the coherent integration time.

Assuming that the signal is ideal and $B_{L} T_{i}$ is small enough, (16) in the limit defined as $d$ is vanishingly small and becomes

$$
\begin{aligned}
\lim _{d \rightarrow 0} \sigma_{\varepsilon}^{2} \cong \sigma_{\mathrm{CRB}}^{2} & \\
& =\frac{B_{L} \int_{-B / 2}^{B / 2} G(f)(\pi f d)^{2} d f}{(2 \pi)^{2}\left(C / N_{0}\right)\left[\int_{-B / 2}^{B / 2} \pi f^{2} d G(f) d f\right]^{2}} \\
& =\frac{B_{L} \pi^{2} d^{2} \int_{-B / 2}^{B / 2} f^{2} G(f) d f}{(2 \pi)^{2}\left(C / N_{0}\right) \pi^{2} d^{2}\left[\int_{-B / 2}^{B / 2} f^{2} G(f) d f\right]^{2}} \\
& =\frac{B_{L}}{(2 \pi)^{2}\left(C / N_{0}\right) \int_{-B / 2}^{B / 2} f^{2} G(f) d f}
\end{aligned}
$$

with

$$
\Delta f_{\text {Gabor }}=\sqrt{\int_{-B / 2}^{B / 2} f^{2} G(f) d f}
$$

where $\sigma_{\mathrm{CRB}}^{2}$ and $\Delta f_{\mathrm{Gabor}}$ are referred to as the Cramér-Rao lower bound and Gabor bandwidth, respectively.

From (17), it is obvious that the Gabor bandwidth can be approximately interpreted as Cramér-Rao lower bound and the greater the Gabor bandwidth, the better the code tracking accuracy.

4.2. Multipath Error Envelopes. The multipath errors are one of the dominant error sources in GNSS. The multipath error envelopes and average multipath errors are valuable indexes to evaluate the multipath mitigation ability. The received baseband signals disturbed by other reflected signals can be expressed as follows [20]:

$$
r(t)=a_{0} e^{j \psi_{0}} x\left(t-\tau_{0}\right)+\sum_{n-1}^{N} a_{n} e^{j \psi_{n}} x\left(t-\tau_{n}\right),
$$

where $a_{0}, \tau_{0}$, and $\psi_{0}$ are the amplitude, delay, and phase of the direct signal. Likewise, $a_{n}, \tau_{n}$, and $\psi_{n}$ are the amplitude, delay, and phase of reflected signals, and $N$ denotes the number of reflected signals. If we only consider one reflected path and use a coherent EML discriminator, the discriminator output can be described as follows [21]:

$$
\begin{aligned}
D(\varepsilon)= & a_{0}\left[\Re\left(\varepsilon-\frac{d}{2}\right)-\mathfrak{R}\left(\varepsilon+\frac{d}{2}\right)\right] \\
& +a_{1}\left[\Re\left(\varepsilon-\Delta \tau-\frac{d}{2}\right)-\Re\left(\varepsilon-\Delta \tau+\frac{d}{2}\right)\right] \\
& \times \cos (\Delta \psi) \equiv 0,
\end{aligned}
$$

where $\Delta \tau$ and $\Delta \psi$ are the delay and carrier phase difference between the multipath and direct signals with $\Delta \tau=\tau_{1}-\tau_{0}$ and $\Delta \psi=\psi_{1}-\psi_{0}$ separately, and $\mathfrak{R}(\cdot)$ and $\varepsilon$ denote autocorrelation function of the signal and the multipath errors, respectively. To explore the theoretical lower bound of the multipath errors, the cases $\Delta \psi=0$ and $\Delta \psi=\pi$ corresponding to the worst multipath errors are considered. By the definition of the Maclaurin series, (20) can be simplified as

$$
D(\varepsilon) \approx D(0)+D^{\prime}(0) \varepsilon .
$$

According to the Wiener-Khintchine theorem, $D(0)$ and $D^{\prime}(0)$ can be obtained by substituting " 0 " into (20) and the corresponding first-order derivative; that is,

$$
\begin{aligned}
& D(0)= \pm 2 a_{1} \int_{-B / 2}^{B / 2} G(f) \sin (-2 \pi f \Delta \tau) \sin (\pi f d) d f \\
& D^{\prime}(0) \\
& \quad=4 \pi a_{0} \int_{-B / 2}^{B / 2} f G(f) \sin (\pi f d) d f \\
& \quad \pm 4 \pi a_{1} \int_{-B / 2}^{B / 2} f G(f) \cos (-2 \pi f \Delta \tau) \sin (\pi f d) d f
\end{aligned}
$$

By combining (21) (22), the multipath error envelopes can be estimated eventually by

$$
\begin{aligned}
& \varepsilon(\Delta \tau) \\
& \approx \frac{ \pm \tilde{a} \int_{-B / 2}^{B / 2} G(f) \sin (2 \pi f \Delta \tau) \sin (\pi f d) d f}{2 \pi \int_{-B / 2}^{B / 2} f G(f) \sin (\pi f d)[1 \pm \tilde{a} \cos (2 \pi f \Delta \tau)] d f},
\end{aligned}
$$

with $\tilde{a}$ multipath to direct ratio (MDR); namely, $\tilde{a}=a_{1} / a_{0}$.

The corresponding average multipath errors can be given by

$$
\varepsilon_{\mathrm{av}}\left(\Delta \tau^{\prime}\right)=\frac{1}{\Delta \tau^{\prime}} \int_{0}^{\Delta \tau^{\prime}} \frac{\left\|\varepsilon_{0}(\Delta \tau)\right\|+\left\|\varepsilon_{\pi}(\Delta \tau)\right\|}{2} d \Delta \tau,
$$

where $\varepsilon_{0}(\Delta \tau)$ and $\varepsilon_{\pi}(\Delta \tau)$ are the multipath errors under the conditions $\Delta \psi=0$ and $\Delta \psi=\pi$.

4.3. Antijamming. The narrowband-jamming and matchedspectrum-jamming are the main threats to the pseudocode and carrier tracking as well as the demodulation process. In order to effectively evaluate the antijamming ability of 
TABLE 1: The parameters in evaluation test.

\begin{tabular}{lcccccc}
\hline Transmitted bandwidth $/ \mathrm{MHz}$ & $B / \mathrm{MHz}$ & $\mathrm{C} / \mathrm{N}_{0} / \mathrm{dB} \cdot \mathrm{Hz}$ & $\mathrm{MDR} / \mathrm{dB}$ & $\Delta \tau / \mathrm{m}$ & $d / \mathrm{chip}$ & $B_{L} / \mathrm{Hz}$ \\
\hline 20.46 & 20.46 & $20 \sim 40$ & -6 & $0 \sim 300$ & 0.1 & 1 \\
\hline
\end{tabular}

navigation signals against the above interferences, the paper introduces four parameters, including anti-narrowbandjamming merit factor $Q_{\text {DemAJNw }}$ and anti-matched-spectrum-jamming merit factor $Q_{\text {DemAJMs }}$ for the demodulation process, and the anti-narrowband-jamming merit factors $Q_{\text {CTAJNW }}$ and anti-matched-spectrum-jamming merit factor $Q_{\text {CTAJMS }}$ for the code tracking process [22]. They are defined as

$$
\begin{aligned}
Q_{\text {DemAJNW }} & =10 \times \log _{10}\left[\frac{1}{R \times \max \left[G_{s}(f)\right]}\right](\mathrm{dB}), \\
Q_{\text {DemAJMS }} & =10 \times \log _{10}\left[\frac{1}{R \times \int_{-B / 2}^{B / 2} G_{s}^{2}(f) d f}\right](\mathrm{dB}), \\
Q_{\mathrm{CTAJNW}} & =10 \times \log _{10}\left[\frac{\int_{-B / 2}^{B / 2} f^{2} G_{s}(f) d f}{\max \left[f^{2} G_{s}(f)\right]}\right](\mathrm{dB}), \\
Q_{\mathrm{CTAJMS}} & =10 \times \log _{10}\left[\frac{\int_{-B / 2}^{B / 2} f^{2} G_{s}(f) d f}{\int_{-B / 2}^{B / 2} f^{2} G_{s}^{2}(f) d f}\right](\mathrm{dB}),
\end{aligned}
$$

where $G_{s}(f)$ and $R$ are the PSD of the desired signals and symbol rate separately. Also, the greater the merit factors, the stronger the antijamming abilities.

\section{Simulation Results and Analysis}

In order to test the validity of multi- $h$ CPM as a candidate modulation for C-band signals, we employ Monte Carlo simulations to evaluate the candidate's performance in the aspects of tracking accuracy, multipath mitigation, and antijamming. The parameters in all simulations are listed in Table 1; as is mentioned above, $B$ is the receiver prefiltering bandwidth and is equal to $20.46 \mathrm{MHz}, C / N_{0}$ is the carrierto-noise ratio (CNR) in AWGN channel and limited in scope $[20,40] \mathrm{dB} \cdot \mathrm{Hz}$, multipath to direct ratio (MDR) is set as $-6 \mathrm{~dB}, \Delta \tau$ is the delay between the multipath and direct signals and is within $0 \sim 300 \mathrm{~m}$, the correlation time spacing between the early and late reference signals $d$ is 0.1 chip, and tracking loop bandwidth $B_{L}$ is $1 \mathrm{~Hz}$.

Figure 4 displays the Gabor bandwidth of CPM signals with different parameters. As we see from Figure 4, the Gabor bandwidth of CPM signals with RC is larger than others with REC in the same condition of modulation index when $B$ is varied from 0 to $25 \mathrm{MHz}$. If other parameters of CPM signals are identical, the large average of all elements in set $\left\{h_{i}\right\}$ leads to a great Gabor bandwidth. The Gabor bandwidth order is as follows when $B$ is fixed at $20.46 \mathrm{MHz}$ : $\mathrm{RC} / \mathbf{h}=\{1 / 2,3 / 4\}>$ $\mathrm{REC} / \mathbf{h}=\{1 / 2,3 / 4\}>\mathrm{RC} / h=1 / 2>\mathrm{GMSK}>\mathrm{MSK}>\mathrm{RC} / \mathbf{h}=$ $\{1 / 4,1 / 2\}>\operatorname{REC} / \mathbf{h}=\{1 / 4,1 / 2\}$.

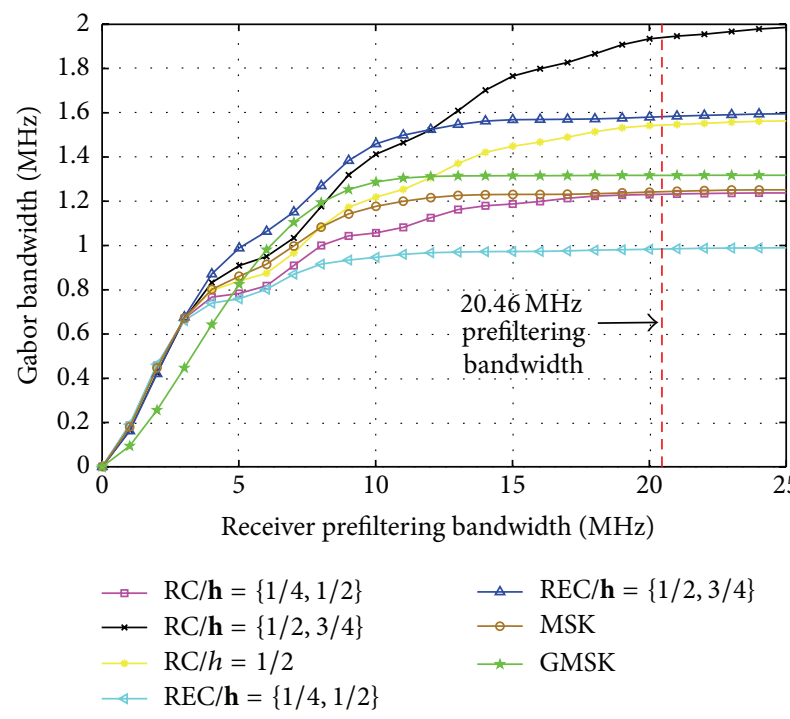

FIGURE 4: The Gabor bandwidth of different CPM signals.

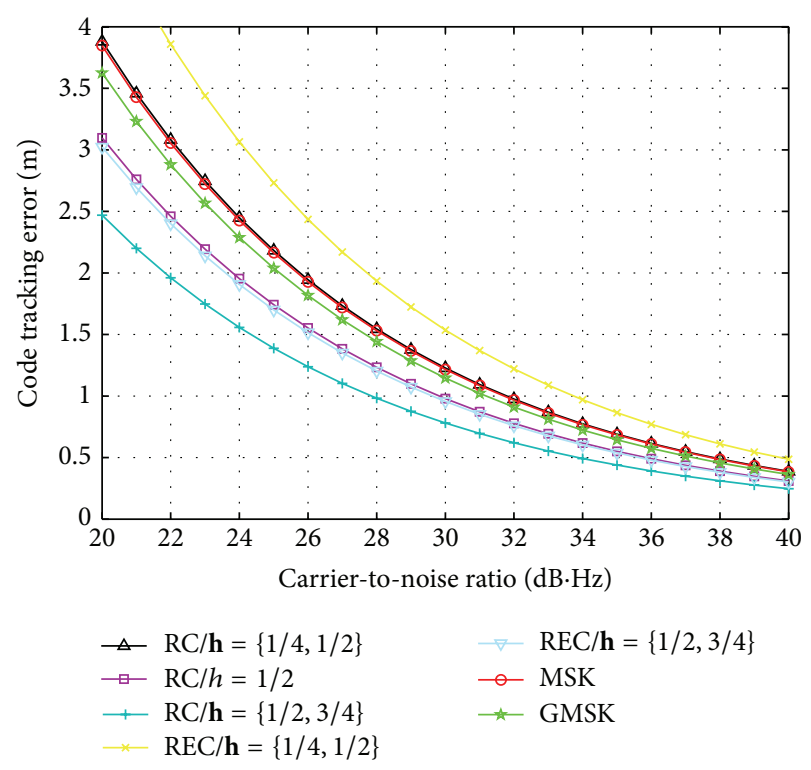

FIgURE 5: The code tracking error of different CPM signals.

The relation curves of code tracking error and $C / N_{0}$ are shown in Figure 5. Obviously, the code tracking error of all CPM schemes is descending with the increasing of $C / N_{0}$ and tends to be stable when $C / N_{0}$ is more than $32 \mathrm{~dB} \cdot \mathrm{Hz}$ and all other parameters are the same as the previous simulation. The code tracking error of CPM signals with RC is relatively small compared to other CPM signals with REC in the same condition of modulation index. In addition, the large average of all elements in set $\left\{h_{i}\right\}$ results in a small code tracking error 


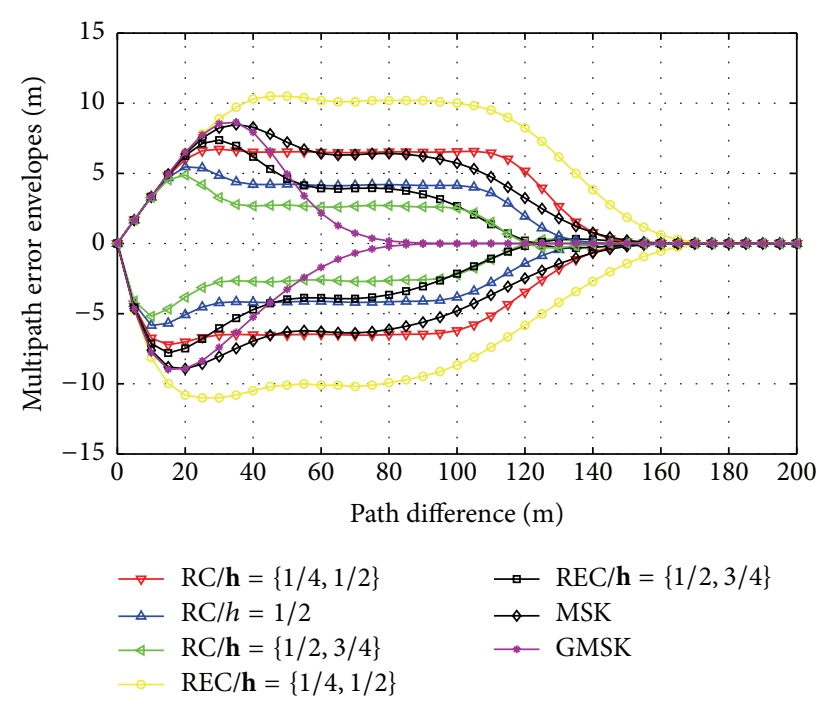

FIGURE 6: The multipath error envelopes of different CPM signals.

if other parameters of CPM signals are identical. The code tracking error order is as follows when $C / N_{0}$ is varied from 20 to $40 \mathrm{~dB} \cdot \mathrm{Hz}: \mathrm{RC} / \mathbf{h}=\{1 / 2,3 / 4\}<\mathrm{REC} / \mathbf{h}=\{1 / 2,3 / 4\}<$ $\mathrm{RC} / h=1 / 2<\mathrm{GMSK}<\mathrm{MSK}<\mathrm{RC} / \mathbf{h}=\{1 / 4,1 / 2\}<\mathrm{REC} / \mathbf{h}=$ $\{1 / 4,1 / 2\}$.

Figure 6 shows the relation curves of multipath error envelopes of different CPM schemes and path difference that is product of $\Delta \tau$ and velocity of light. In Figure 6 we observe that the multipath error of CPM signals with RC is smaller than other CPM signals with REC in the same condition of modulation index when path difference is in the region of 1 to $150 \mathrm{~m}$. Similarly, a great average of all elements in set $\left\{h_{i}\right\}$ tends to be a small multipath error if other parameters of CPM signals are the same. When path difference is varied from 1 to $60 \mathrm{~m}$, the CPM signal with $R C / \mathbf{h}=\{1 / 2,3 / 4\}$ has the best performance of multipath mitigation in the above schemes, and GMSK is superior to other scheme in the region of 60 to $150 \mathrm{~m}$.

Figure 7 shows the comparison of antijamming of CPM signals with different parameters. As we see from Figure 7, in the aspect of $Q_{\text {DemAJNW }}$, GMSK has the best performance in all CPM schemes, and the CPM signal with $\mathrm{RC} / \mathrm{h}=$ $\{1 / 2,3 / 4\}$ has almost the same performance as that with $\mathrm{REC} / \mathbf{h}=\{1 / 2,3 / 4\}$, but both of them are inferior to GMSK. In the aspect of $Q_{\text {CTAJNW }}$, the CPM signal employed $\mathrm{RC} / \mathbf{h}$ $=\{1 / 2,3 / 4\}$ has the best performance in all CPM schemes. In the aspect of $Q_{\text {DemAJMS }}$, the CPM scheme adopted $\mathrm{RC} / \mathbf{h}$ $=\{1 / 2,3 / 4\}$ parameter has better performance than others, inferior to GMSK. In the aspect of $Q_{\text {CTAJMS }}$, the CPM scheme with $\mathrm{RC} / \mathbf{h}=\{1 / 2,3 / 4\}$ has the equivalent performance to GMSK, and both of them are superior to others. After the above analysis, we can obtain that the CPM scheme with $\mathrm{RC} / \mathbf{h}=\{1 / 2,3 / 4\}$ is relatively close to GMSK in terms of antijamming ability. If we continue to rationally adjust modulation index set, there must be an optimized multi- $h$ CPM signal whose antijamming performance is superior to GMSK.

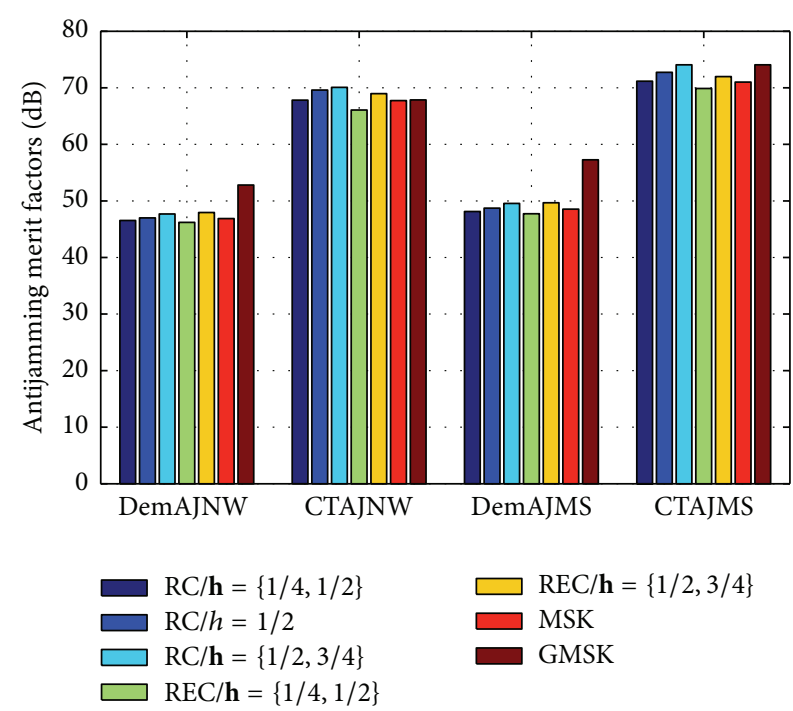

FIGURE 7: The comparison of antijamming of different CPM signals.

\section{Conclusions}

Multi- $h$ CPM signals as a special subclass in CPM family still maintain all of excellent characteristics just as a general CPM, such as constant envelope, high power, and bandwidth efficiency, suitable for adopting HPA. Besides, they also have some unique properties, that is, flexible parameter adjusting, a large number of alternative waveforms, being easily compatible with existing signals, and deployment capabilities of multiband and multifrequency and so on. Therefore, multi$h$ CPM will bring extensive application prospects for future GNSS signals.

According to transmission properties and the constraint condition of compatibility in C-band, multi- $h$ CPM is proposed as a candidate modulation waveform for C-band signals, and a proper tradeoff among channel capacity, band efficiency, navigation performance, and implementation complexity of receiver can be realized by optimizing modulation indexes. Simulation results show that multi- $h$ CPM signals not only provide better performance in terms of tracking accuracy, multipath mitigation, and antijamming compared to MSK, GMSK, and other single- $h$ CPM signals under the constraint on transmitted bandwidth in C-band, but also have relatively high channel capacity and band efficiency while taking into account receiver complexity. Furthermore, the proposed modulation scheme could offer new ideas and feasibility demonstration for signal system design of next generation GNSS.

\section{Conflict of Interests}

The authors declare that there is no conflict of interests regarding the publication of this paper.

\section{Acknowledgments}

This paper is supported by the National Natural Science Foundation of China (Grant no. 61403093), Science 
Foundation of Heilongjiang Province of China for Returned Scholars (Grant no. LC2013C22), the Assisted Project by Heilongjiang Province of China Postdoctoral Funds for Scientific Research Initiation (Grant no. LBH-Q14048), and China Fundamental Research Funds for Central Universities (Grant no. HEUCF1508).

\section{References}

[1] J. W. Betz, "Signal structures for satellite-based navigation: past, present and future," Inside GNSS, vol. 8, pp. 34-42, 2013.

[2] X. Liu, M. Liang, Y. Morton, P. Closas, T. Zhang, and Z. Hong, "Performance evaluation of MSK and OFDM modulations for future GNSS signals," GPS Solutions, vol. 18, no. 2, pp. 163-175, 2014.

[3] E. Colzi, G. Lopez-Risueño, J. Samson et al., "Assessment of the feasibility of GNSS in C-band," in Proceedings of the 26th AIAA International Communications Satellite Systems Conference (ICSSC '08), pp. 1-15, June 2008.

[4] M. Liu, X. Zhan, W. Li, and M. Chen, "MSK-BCS modulation for GNSS radio frequency compatibility in C band," Journal of Networks, vol. 9, no. 10, pp. 2713-2720, 2014.

[5] I. Mateu, M. Paonni, and J. L. Issler, "A search for spectrumGNSS signals in S band," Inside GNSS, vol. 5, no. 7, pp. 46-53, 2010.

[6] J.-L. Issler, M. Paonni, and B. Eissfeller, "Toward centimetric positioning thanks to L- and S-band GNSS and to meta-GNSS signals," in Proceedings of the 5th ESA Workshop on Satellite Navigation Technologies and European Workshop on GNSS Signals and Signal Processing (NAVITEC '10), pp. 1-8, December 2010.

[7] P. Qing, "The research of the signal in the S frequency band," in Proceedings of the China Satellite Navigation Conference (CSNC '13), vol. S2, pp. 1-5, May 2013.

[8] M. Liu, X. Zhan, W. Li, and M. Chen, "A compatibility analysis between GNSS and radio astronomy/microwave landing system in C band," Journal of Aeronautics, Astronautics and Aviation, vol. 46, no. 2, pp. 102-107, 2014.

[9] X.-L. Hu, Y.-H. Ran, Y.-Q. Liu, and T. Ke, "New option for modulation in GNSS signal design," Systems Engineering and Electronics, vol. 32, no. 9, pp. 1962-1967, 2010.

[10] S. Attia, K. Rouabah, D. Chikouche, and M. Flissi, "Side peak cancellation method for sine-BOC $(m, n)$-modulated GNSS signals," EURASIP Journal on Wireless Communications and Networking, vol. 2014, article 34, 2014.

[11] J. A. Avila-Rodriguez, S. Wallner, J. H. Won, and B. Eissfeller, "Study on a Galileo signal and service plan for C-band," in Proceedings of the 21th International Technical Meeting of the Satellite Division, pp. 2515-2530, September 2008.

[12] X. Gu, R. Li, D. Zeng, and D. Yao, "Study on MSK modulation and tracking technique for satellite navigation systems," in Proceedings of the IET International Radar Conference, April 2013.

[13] M. Luise, "Easy calculation of power spectra for multi-h phasecoded signals," Electronics Letters, vol. 21, no. 14, pp. 608-609, 1985.

[14] S. Zhong, C. Yang, and J. Zhang, "Symbol timing estimation with multi-h CPM signals," Journal of Networks, vol. 9, no. 4, pp. 921-926, 2014.

[15] R. Chen, F. Wei, M. Huang, B. Li, and B. Bai, "On the capacity of CPM over Rayleigh fading channels," in Proceedings of the International Conference on Wireless Communications and Signal Processing (WCSP '12), pp. 1-15, Huangshan, China, October 2012.

[16] D. M. Arnold, H.-A. Loeliger, P. O. Vontobel, A. Kavcic, and W. Zeng, "Simulation-based computation of information rates for channels with memory," IEEE Transactions on Information Theory, vol. 52, no. 8, pp. 3498-3508, 2006.

[17] S. Cheng, M. C. Valenti, and D. Torrieri, "Coherent continuousphase frequency-shift keying: parameter optimization and code design," IEEE Transactions on Wireless Communications, vol. 8, no. 4, pp. 1792-1802, 2009.

[18] Z. Tang, H. Zhou, and X. Hu, "Research on performance evaluation of COMPASS signal," Scientia Sinica: Physica, Mechanica \& Astronomica, vol. 5, pp. 592-602, 2010.

[19] J. W. Betz and K. R. Kolodziejski, "Generalized theory of code tracking with an early-late discriminator. Part I. Lower bound and coherent processing," IEEE Transactions on Aerospace and Electronic Systems, vol. 45, no. 4, pp. 1538-1556, 2009.

[20] M. Sahmoudi and R. J. Landry, "Multipath mitigation techniques using maximum likelihood principle," Inside GNSS, vol. 8, pp. 24-29, 2008.

[21] E. D. Kaplan and C. J. Hegarty, Understanding GPS: Principle and Application, Artech House, Boston, Mass, USA, 2006.

[22] R. Xue, Y. Sun, and D. Zhao, "CPM signals for satellite navigation in the S and C bands," Sensors, vol. 15, no. 6, pp. 1318413200, 2015. 


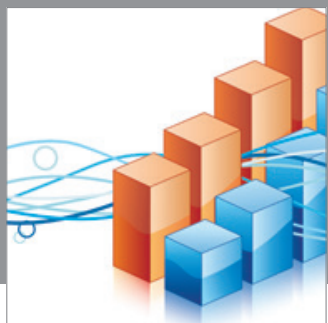

Advances in

Operations Research

mansans

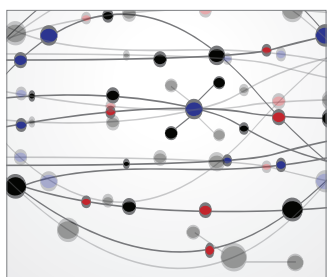

The Scientific World Journal
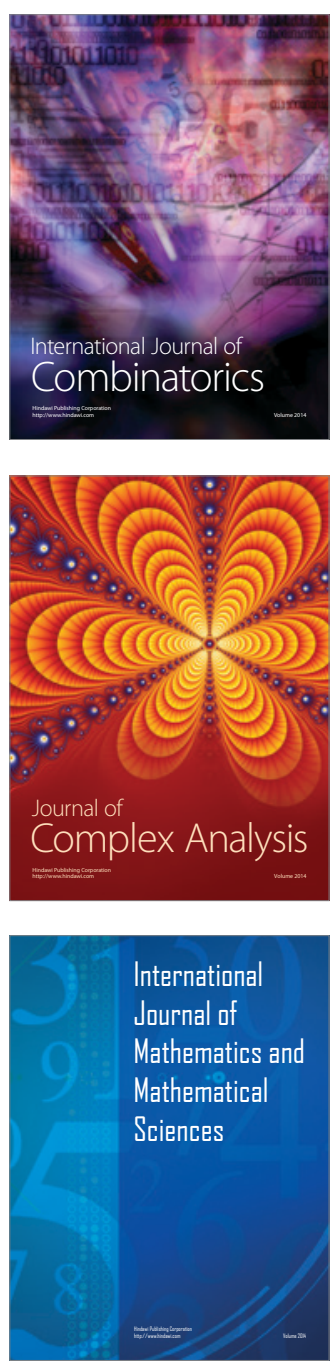
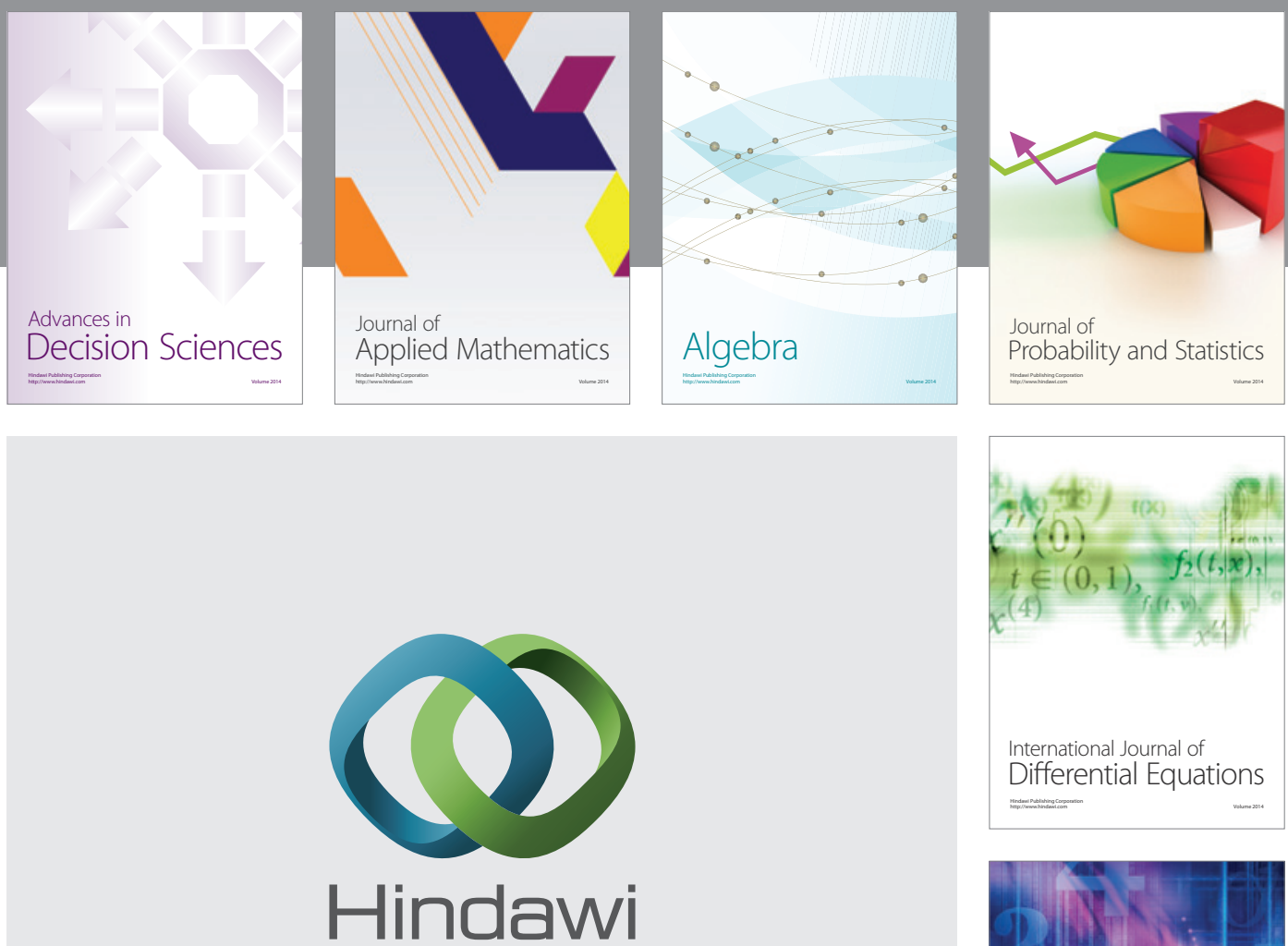

Submit your manuscripts at http://www.hindawi.com
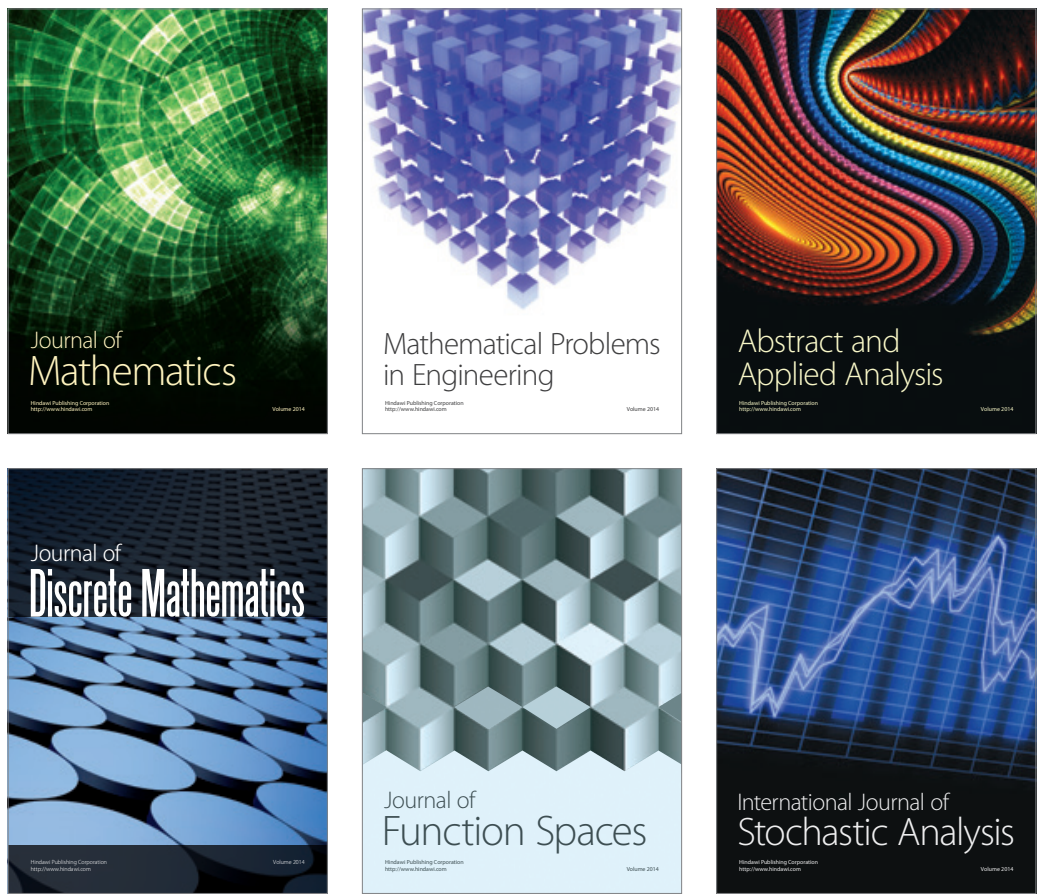

Journal of

Function Spaces

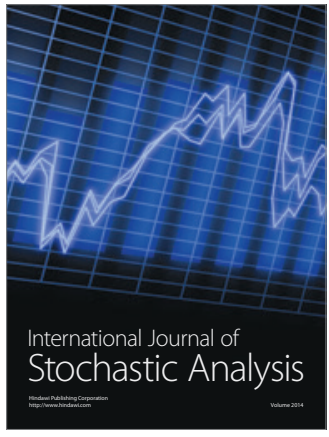

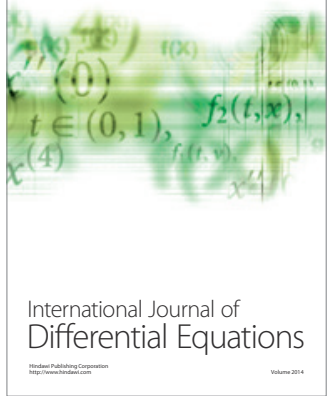
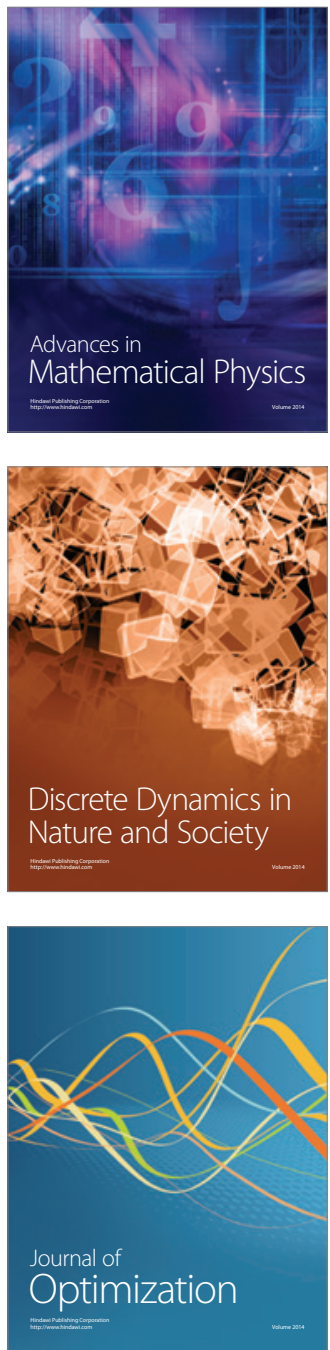